\title{
ON THE DIVERGENCE OF LAGRANGE INTERPOLATION WITH EQUIDISTANT NODES
}

\author{
X. LI AND R. N. MOHAPATRA
}

(Communicated by J. Marshall Ash)

\begin{abstract}
This paper is concerned with the optimal rate of divergence of Lagrange interpolation of $f(x)=|x|$ at equidistant nodes.
\end{abstract}

\section{MAin Results}

In this note we discuss a problem in the divergent aspect of Lagrange interpolation. Denote $x_{k, n}:=-1+2(k-1) /(n-1), k=1,2, \ldots, n, n=2,3, \ldots$. Recall that given a function $f(x)$ defined on $[-1,1]$, the Lagrange interpolation polynomial $L_{n}(f ; x)$, of degree at most $n-1$, is (uniquely) defined by the conditions

$$
L_{n}\left(f ; x_{k, n}\right)=f\left(x_{k, n}\right) \quad(k=1,2, \ldots, n ; n=2,3, \ldots) .
$$

The following divergent result of Bernstein is well known (cf. [N, p. 30]).

Theorem 1 (Bernstein, 1918). For function $f(x)=|x|$, the sequence $\left\{L_{n}(f ; x)\right.$ : $n=2,3, \ldots\}$ diverges if $0<|x|<1$.

Recently, Byrne, Mills, and Smith [BMS] considered the rate of this divergence process. More precisely, they proved

Theorem 2 (Byrne, Mills, and Smith, 1990 [BMS]). For the function $f(x)=|x|$, we have

$$
\limsup _{n \rightarrow \infty} n^{-1} \log \left|L_{n}(f ; x)-\right| x||=\frac{1}{2}[(1+x) \log (1+x)+(1-x) \log (1-x)]
$$

if $0<|x|<1$.

From now on, we will write $L_{n}(x)=L_{n}(f ; x)$ if $f(x)=|x|$.

The result of Byrne, Mills, and Smith tells us that for every $x$ with $0<$ $|x|<1$, there exists a subsequence, say $\left\{L_{n}(x): n=n_{1}, n_{2}, n_{3}, \ldots\right\}$, of $\left\{L_{n}(x): n=2,3, \ldots\right\}$, whose rate of divergence is geometrically fast; but it seems that the sequence $\left\{n_{1}, n_{2}, n_{3}, \ldots\right\}$ should depend on $x$. We will show that there actually exists a subsequence that works for almost all $x$ (with $0<|x|<1$ ) as implied by the following results.

Received by the editors March 26, 1991 and, in revised form, July 16, 1991, October 10, 1991, and January 10, 1992.

1991 Mathematics Subject Classification. Primary 41A05.

Key words and phrases. Divergence, Lagrange interpolation. 
Theorem 3. For all $x \in R$, we have

$$
\lim _{n \rightarrow \infty}\left|\frac{L_{n}(x)-|x|}{W_{n}(x)}\right|^{1 / n}=e,
$$

where $W_{n}(x):=\prod_{k=1}^{n}\left(x-x_{k, n}\right)$.

Corollary 4. Let $\left\{p_{k}: k=1,2,3, \ldots\right\}$ be the sequence of all positive prime integers with $p_{1}<p_{2}<p_{3}<\cdots$. Then for $0<|x|<1$, we have

$$
\begin{aligned}
\limsup _{n \rightarrow \infty} n^{-1} \log \left|L_{n}(x)-\right| x|| & =\limsup _{k \rightarrow \infty}\left(p_{k}+1\right)^{-1} \log \left|L_{p_{k}+1}(x)-\right| x|| \\
& =\frac{1}{2}[(1+x) \log (1+x)+(1-x) \log (1-x)] .
\end{aligned}
$$

Furthermore, if we denote $T:=\left\{x \in[-1,1]\left|\liminf _{n \rightarrow \infty} \min _{1 \leq k \leq n}\right| x-\left.x_{k, n}\right|^{1 / n}\right.$ $<1\}$, then $T$ is of Lebesgue measure zero and for $x \in[-1,1] \backslash T$,

(2) $\lim _{k \rightarrow \infty}\left(p_{k}+1\right)^{-1} \log \left|L_{p_{k}+1}(x)-\right| x||=\frac{1}{2}[(1+x) \log (1+x)+(1-x) \log (1-x)]$.

Remark. Such a set $T$ is not empty. A number $x^{*}$ in $T$ can be constructed as follows. Set $10^{\langle 1\rangle}:=10,10^{\langle 2\rangle}:=10^{10^{\langle 1\rangle}}, \ldots, 10^{\langle k+1\rangle}=10^{10^{\langle k\rangle}}, \ldots$ Then

$$
x^{*}=\frac{1}{10^{\langle 1\rangle}}-\frac{2}{10^{\langle 2\rangle}}+\cdots+\frac{1}{10^{\langle 2 k-1\rangle}}-\frac{2}{10^{\langle 2 k\rangle}}+\cdots
$$

is a number in $(0,1)$. It is easy to see that if $n_{j}:=10^{\langle 2 j\rangle}+1$, then

$$
x_{k\left(n_{j}\right), n_{j}}=\frac{1}{10^{\langle 1\rangle}}-\frac{2}{10^{\langle 2\rangle}}+\cdots+\frac{1}{10^{\langle 2 j-1\rangle}}-\frac{2}{10^{\langle 2 j\rangle}}
$$

and

So

$$
0<x^{*}-x_{k\left(n_{j}\right), n_{j}}<\frac{1}{10^{\langle 2 j+1\rangle}}
$$

$$
\left|x^{*}-x_{k\left(n_{j}\right), n_{j}}\right|^{1 /\left(n_{j}-1\right)}<\frac{1}{10}
$$

thus $x^{*} \in T$.

We shall assume Theorem 3 for the moment and prove Corollary 4 . The proof of Theorem 3 will be given in $\S 2$.

We need the following elementary result for the proof of Corollary 4.

Lemma 5. Let $W_{n}(x)$ be as in Theorem 3 and $T$ as in Corollary 4 . If $x$ is an irrational number in $[-1,1] \backslash T$ or a real number in $(-\infty,-1) \cup(1, \infty)$, then

$$
\lim _{n \rightarrow \infty}\left|W_{n}(x)\right|^{1 / n}=e^{-1}|1+x|^{(1+x) / 2}|1-x|^{(1-x) / 2} .
$$

If $x$ is a rational number in $[-1,1]$, define

$$
\Lambda_{x}:=\{n: n=1,2,3, \ldots \text { and } n+x(n-1) \text { is an odd integer }\}
$$

then

$$
W_{n}(x)=0 \quad\left(n \in \Lambda_{x}\right)
$$

and

(4) $\quad \limsup _{n \rightarrow \infty}\left|W_{n}(x)\right|^{1 / n}=\lim _{\substack{n \rightarrow \infty \\ n \notin \Lambda_{x}}}\left|W_{n}(x)\right|^{1 / n}=e^{-1}(1+x)^{(1+x) / 2}(1-x)^{(1-x) / 2}$. 
This result may be well known. We include a proof for the sake of completeness. For relevant discussions, see [D, p. 84; IK, Chapter 6, §3.6].

Proof of Lemma 5. First, if $x$ is a real number in $(-\infty,-1) \cup(1, \infty)$, then $\log |x-t|$ is a continuous function of $t$ over the interval $[-1,1]$ and

$$
\frac{1}{n-1} \log \left|W_{n}(x)\right|-\frac{1}{n-1} \log |x-1|=\frac{1}{n-1} \sum_{k=1}^{n-1} \log \left|x-x_{k, n}\right|
$$

is just a Riemann sum of the integral $\frac{1}{2} \int_{-1}^{1} \log |x-t| d t$; thus

$$
\lim _{n \rightarrow \infty} \frac{1}{n-1} \log \left|W_{n}(x)\right|=\frac{1}{2} \int_{-1}^{1} \log |x-t| d t .
$$

Since

$$
\frac{1}{2} \int_{-1}^{1} \log |x-t| d t=\frac{1}{2}[(1+x) \log |1+x|+(1-x) \log |1-x|]-1
$$

for all $x \in \mathbf{R}$, it follows that (5) implies (3).

Next, when $x$ is an irrational number in $[-1,1]$, we show

$$
\lim _{n \rightarrow \infty} \frac{1}{n} \sum_{k=1}^{k(n)-1} \log \left(x-x_{k, n}\right)=\frac{1}{2} \int_{-1}^{x} \log (x-t) d t
$$

and

$$
\lim _{n \rightarrow \infty} \frac{1}{n} \sum_{k=k(n)+2}^{n} \log \left(x_{k, n}-x\right)=\frac{1}{2} \int_{x}^{1} \log (t-x) d t,
$$

where $k(n):=\max \left\{k: k \geq 1\right.$ and $\left.x_{k, n}<x\right\}$. (The dependence of $k(n)$ on $x$ is omitted for simplicity of notation.)

We only prove (6), the proof of (7) is similar.

Let the irrational $x \in[-1,1]$ be fixed. Since $\log (x-t)$ is a decreasing function of $t$ for $t<x$, we have

$$
\frac{1}{2} \int_{x_{k-1, n}}^{x_{k, n}} \log (x-t) d t<\frac{1}{n-1} \log \left(x-x_{k-1, n}\right)
$$

for all $k \leq k(n)$ and $k \geq 2$. Summing both sides for $k=2,3, \ldots, k(n)$, we get

$$
\frac{1}{2} \sum_{k=2}^{k(n)} \int_{x_{k-1, n}}^{x_{k, n}} \log (x-t) d t<\frac{1}{n-1} \sum_{k=2}^{k(n)} \log \left(x-x_{k-1, n}\right) .
$$

Since the left side equals $\frac{1}{2} \int_{-1}^{x_{k(n), n}} \log (x-t) d t$ and $x_{k(n), n} \rightarrow x$ as $n \rightarrow \infty$, the existence of the improper integral $\int_{-1}^{x} \log (x-t) d t$ implies that the left side of (8) tends to $\frac{1}{2} \int_{-1}^{x} \log (x-t) d t$ as $n \rightarrow \infty$. Thus, letting $n \rightarrow \infty$ in (8), we get

$$
\frac{1}{2} \int_{-1}^{x} \log (x-t) d t \leq \liminf _{n \rightarrow \infty} \frac{1}{n-1} \sum_{k=1}^{k(n)-1} \log \left(x-x_{k, n}\right) .
$$


On the other hand, we have

$$
\frac{1}{n-1} \log \left(x-x_{k, n}\right)<\frac{1}{2} \int_{x_{k-1, n}}^{x_{k, n}} \log (x-t) d t .
$$

Summing both sides for $k=2,3, \ldots, k(n)-1$ and using arguments similar to those used to prove (9), we obtain

$$
\limsup _{n \rightarrow \infty} \frac{1}{n-1} \sum_{k=2}^{k(n)-1} \log \left(x-x_{k, n}\right) \leq \frac{1}{2} \int_{-1}^{x} \log (x-t) d t .
$$

From (9) and (10), equality (6) follows.

Now, if irrational $x$ is not in $T$, since we have

$$
\frac{2}{n-1} \log \left(\min _{1 \leq k \leq 1}\left|x-x_{k, n}\right|\right) \leq \frac{1}{n-1} \log \left|\left(x-x_{k(n), n}\right)\left(x-x_{k(n)+1, n}\right)\right| \leq 0,
$$

then

$$
\lim _{n \rightarrow \infty} \frac{1}{n-1} \log \left|\left(x-x_{k(n), n}\right)\left(x-x_{k(n)+1, n}\right)\right|=0 .
$$

Applying (6), (7), and (11), we conclude (3).

We have proved the first half of the lemma. Next, we assume $x$ is a rational number in $[-1,1]$. If $n \in \Lambda_{x}$, then

$$
x=x_{(n+x(n-1)+1) / 2, n},
$$

hence

$$
W_{n}(x)=W_{n}\left(x_{(n+x(n-1)+1) / 2, n}\right)=0 .
$$

Thus the first equality in (4) follows if we can prove the second equality. If $n \notin \Lambda_{x}$, then $x \neq x_{k, n}, k=1,2, \ldots, n, n=1,2,3, \ldots$. Since numbers in $T \backslash\{-1,0,1\}$ must be transcendental by Liouville's theorem (cf. [HW, Theorem 191, p. 161]), we also have $x \notin T$. So (11) still holds. Then, similar to the proof of $(6)$, we can show

$$
\lim _{\substack{n \rightarrow \infty \\ n \notin \Lambda_{x}}} \frac{1}{n} \sum_{k=1}^{k(n)-1} \log \left(x-x_{k, n}\right)=\frac{1}{2} \int_{-1}^{x} \log (x-t) d t
$$

and

$$
\lim _{\substack{n \rightarrow \infty \\ n \notin \Lambda_{x}}} \frac{1}{n} \sum_{k=k(n)+2}^{n} \log \left(x_{k, n}-x\right)=\frac{1}{2} \int_{x}^{1} \log (t-x) d t
$$

which, together with (11), imply the second equality in (4).

Proof of Corollary 4. From equality (3), for irrational $x \in[-1,1] \backslash T$, $\lim _{n \rightarrow \infty}\left|W_{n}(x)\right|^{1 / n}$ exists; so $\lim _{k \rightarrow \infty}\left|W_{p_{k}+1}(x)\right|^{1 /\left(p_{k}+1\right)}$ exists and is equal to $\lim _{n \rightarrow \infty}\left|W_{n}(x)\right|^{1 / n}$, i.e.,

(12) $\lim _{n \rightarrow \infty}\left|W_{n}(x)\right|^{1 / n}=\lim _{k \rightarrow \infty}\left|W_{p_{k}+1}(x)\right|^{1 /\left(p_{k}+1\right)}=\frac{1}{e}(1+x)^{(1+x) / 2}(1-x)^{(1-x) / 2}$.

Now, for rational $x$ with $0<|x|<1$, say $x=p / q$ with $(p, q)=1$ and $q>1$, note that

$$
n \in \Lambda_{x} \text { implies }(n-1) / q \text { is an integer ; }
$$


we conclude that $\left\{p_{k}+1: p_{k}>q\right\} \cap \Lambda_{x}=\varnothing$. Then (4) implies

(13) $\lim _{k \rightarrow \infty}\left|W_{p_{k}+1}(x)\right|^{1 /\left(p_{k}+1\right)}=\lim _{\substack{n \rightarrow \infty \\ n \notin \Lambda_{x}}}\left|W_{n}(x)\right|^{1 / n}=\frac{1}{e}(1+x)^{(1+x) / 2}(1-x)^{(1-x) / 2}$.

Combining (12) and (13), by Theorem 3 we obtain (2).

To prove (1), in view of Theorem 3 and equations (12) and (13), it suffices to show (no matter whether $x \in T$ or not)

$$
\begin{aligned}
\limsup _{n \rightarrow \infty}\left|W_{n}(x)\right|^{1 / n} & =\underset{k \rightarrow \infty}{\limsup \left|W_{p_{k}+1}(x)\right|^{1 /\left(p_{k}+1\right)}} \\
& =\frac{1}{e}(1+x)^{(1+x) / 2}(1-x)^{(1-x) / 2}, \quad x \in(-1,1) .
\end{aligned}
$$

Note that for $x \in(-1,1)$ and $n$ large enough,

$$
\frac{1}{n} \log \left|W_{n}(x)\right| \leq \frac{1}{n} \log \left|\frac{W_{n}(x)}{\left(x-x_{k(n), n}\right)\left(x-x_{k(n)+1, n}\right)}\right| .
$$

Using (6) and (7), the left side tends to $\frac{1}{2} \int_{-1}^{1} \log |x-t| d t$. So letting $n \rightarrow \infty$ gives

$$
\limsup _{n \rightarrow \infty} \frac{1}{n} \log \left|W_{n}(x)\right| \leq \frac{1}{2} \int_{-1}^{1} \log |x-t| d t .
$$

We need only to show

$$
\limsup _{k \rightarrow \infty} \frac{1}{p_{k}+1} \log \left|W_{p_{k}+1}(x)\right| \geq \frac{1}{2} \int_{-1}^{1} \log |x-t| d t .
$$

In fact, since (6) and (7) hold with $p_{k}+1$ replacing $n$, we only have to show

$$
\limsup _{n=p_{j}+1 \rightarrow \infty} \frac{1}{n} \log \left|\left(x-x_{k(n), n}\right)\left(x-x_{k(n)+1, n}\right)\right|=0, \quad|x|<1 .
$$

If (14) is false, then there exists $r<0$ such that, for $j$ large enough,

$$
\frac{1}{n} \log \left|\left(x-x_{k(n), n}\right)\left(x-x_{k(n)+1, n}\right)\right|<r<0, \quad n=p_{j}+1 .
$$

Assume $\left|x-x_{h(n), n}\right|=\min _{1 \leq k \leq n}\left|x-x_{k, n}\right|$; then $h(n)=k(n)$ or $k(n)+1$ and

$$
\left|\left(x-x_{k(n), n}\right)\left(x-x_{k(n)+1, n}\right)\right| \geq \frac{1}{2(n-1)}\left|x-x_{h(n), n}\right| \text {. }
$$

Using this inequality in (15), we have, for some $\rho \in(0,1)$ and $j$ large enough,

$$
\left|x-x_{h(n), n}\right|<\rho^{n}, \quad n=p_{j}+1 \text {. }
$$

So

$$
\left|x_{h(n), n}-x_{h\left(n^{\prime}\right), n^{\prime}}\right| \leq\left|x-x_{h(n), n}\right|+\left|x-x_{h\left(n^{\prime}\right), n^{\prime}}\right|<2 \rho^{n}
$$

with $n=p_{j}+1$ and $n^{\prime}=p_{j+1}+1$. Denote $s_{j}=h(n)-1$ and $s_{j+1}=h\left(n^{\prime}\right)-1$. Then (16) can be written as

or, equivalently,

$$
\left|\frac{2 s_{j}}{p_{j}}-\frac{2 s_{j+1}}{p_{j+1}}\right|<2 \rho^{p_{j}}
$$

$$
\left|s_{j} p_{j+1}-s_{j+1} p_{j}\right|<p_{j} p_{j+1} \rho^{p_{j}} \text { for } j \text { large enough. }
$$


Now using the famous Bertrand's Postulate in number theory (cf. [HW, Theorem 418 , p. 343]), we know $p_{j+1}<2 p_{j}$. So

$$
\left|s_{j} p_{j+1}-s_{j+1} p_{j}\right|<2 p_{j}^{2} \rho^{p_{j}} \rightarrow 0 \text { as } j \rightarrow \infty .
$$

Since $s_{j} p_{j+1}-s_{j+1} p_{j}$ is an integer, it follows that

$$
s_{j} p_{j+1}-s_{j+1} p_{j}=0 \text { for } j \text { large enough. }
$$

But this is absurd because $0<s_{j}<p_{j}$ and $0<s_{j+1}<p_{j+1}$ for $j$ large enough and both $p_{j}$ and $p_{j+1}$ are primes. So (14) must be true, thus (1) holds.

Finally, we prove $T$ is of Lebesgue measure zero. Note that

$$
T=\bigcup_{m=1}^{\infty} \bigcap_{j=1}^{\infty} \bigcup_{n=j}^{\infty} \bigcup_{k=1}^{n}\left(x_{k, n}-\left(1-\frac{1}{m}\right)^{n}, x_{k, n}+\left(1-\frac{1}{m}\right)^{n}\right) \text {. }
$$

Let $\mu^{*}$ denote the Lebesgue outer measure. Then

$$
\begin{aligned}
\mu^{*}(T) & \leq \sum_{m=1}^{\infty} \mu^{*}\left(\varlimsup_{n \rightarrow \infty} \bigcup_{k=1}^{n}\left(x_{k, n}-\left(1-\frac{1}{m}\right)^{n}, x_{k, n}+\left(1-\frac{1}{m}\right)^{n}\right)\right) \\
& =\sum_{m=1}^{\infty} \limsup _{n \rightarrow \infty} \mu^{*}\left(\bigcup_{k=1}^{n}\left(x_{k, n}-\left(1-\frac{1}{m}\right)^{n}, x_{k, n}+\left(1-\frac{1}{m}\right)^{n}\right)\right) \\
& \leq \sum_{m=1}^{\infty} \limsup _{n \rightarrow \infty} 2 n\left(1-\frac{1}{m}\right)^{n}=0 .
\end{aligned}
$$

This completes the proof of Corollary 4 .

Before we prove Theorem 3, we would like to remark that a closer look at the proof of Theorem 1 given in [BMS] would suggest a possible proof of Theorem 3, but their proof uses Lagrange interpolation formula and involves a tricky transformation and hypergeometric series identities, which is entirely different from Bernstein's approach of using Newton's interpolation formula. As another goal of this note we present a short and elementary proof of Theorem 3 by using Bernstein's approach.

Recall that the Lagrange interpolation polynomial $L_{n}(f ; x)$ can be expressed by Newton's formula (cf. [D, N])

$$
L_{n}(f ; x)=\sum_{k=0}^{n-1}\left(\frac{n-1}{2}\right)^{k} \frac{\Delta_{n}^{k} f(-1)}{k !}\left(x-x_{1, n}\right) \cdots\left(x-x_{k, n}\right),
$$

where

$$
\Delta_{n}^{l} f(-1)=\sum_{r=0}^{l}(-1)^{l-r}\left(\begin{array}{l}
l \\
r
\end{array}\right) f\left(x_{r+1, n}\right), \quad l=0,1, \ldots, n-1 .
$$

\section{Proof of Theorem 3}

We prove the theorem for $x$ such that $x<0$; the result when $0<x$ can then be obtained by symmetry. Denote $n^{\prime}:=[(n-1) / 2]$, where $[t]$ denotes the largest integer $\leq t$. If

$$
\phi(t)= \begin{cases}0 & \text { for } t \leq 0, \\ t & \text { for } 0 \leq t,\end{cases}
$$


then

$$
\frac{L_{n}(x)-|x|}{2}=\frac{L_{n}(x)+x}{2}=L_{n}(\phi ; x)
$$

since $x=L_{n}(t ; x)$. Now from Newton's formula we can get

$$
\begin{aligned}
L_{n}(\phi ; x)=\sum_{m=1}^{n^{\prime}} & (-1)^{m-1}\left(\frac{n-1}{2}\right)^{n^{\prime}+m} \\
& \times \frac{\left[1+\left(n-1-2 n^{\prime}\right)\left(n^{\prime}-m\right)\right]\left(x-x_{1}\right)\left(x-x_{2}\right) \cdots\left(x-x_{n^{\prime}+m}\right)}{(m-1) ! n^{\prime} !\left(n^{\prime}+m\right)\left(n^{\prime}+m-1\right)}
\end{aligned}
$$

In fact, when $n$ is odd, (13) is established as formula (70) of [N, p. 31]. The proof of (17) when $n$ is even is entirely analogous. Next, write (17) as

$$
\begin{aligned}
L_{n}(\phi ; x)=(-1) & \frac{\left(x-x_{1}\right) \cdots\left(x-x_{n^{\prime}}\right)}{n^{\prime} !} \\
& \times \sum_{m=1}^{n^{\prime}}\left(\frac{n-1}{2}\right)^{n^{\prime}+m} \\
& \quad \times \frac{\left[1+\left(n-1-2 n^{\prime}\right)\left(n^{\prime}-m\right)\right]\left(x_{n^{\prime}+1}-x\right) \cdots\left(x_{n^{\prime}+m}-x\right)}{(m-1) !\left(n^{\prime}+m\right)\left(n^{\prime}+m-1\right)} ;
\end{aligned}
$$

then every term in the sum is positive. So

(18) $\left|L_{n}(\phi ; x)\right| \geq \frac{\left|\left(x-x_{1}\right) \cdots\left(x-x_{n^{\prime}}\right)\right|}{n^{\prime} !} \cdot\left(\frac{n-1}{2}\right)^{2 n^{\prime}} \frac{\left(x_{n^{\prime}+1}-x\right) \cdots\left(x_{2 n^{\prime}}-x\right)}{\left(n^{\prime}-1\right) ! 2 n^{\prime}\left(2 n^{\prime}-1\right)}$

and

$$
\begin{aligned}
& \left|L_{n}(\phi ; x)\right| \leq \frac{\left|\left(x-x_{1}\right) \cdots\left(x-x_{n^{\prime}}\right)\right|}{n^{\prime} !} \\
& \times \sum_{m=1}^{n^{\prime}}\left(\frac{n-1}{2}\right)^{n^{\prime}+m} \\
& \times \frac{n^{\prime}\left(x_{n^{\prime}+1}-x\right) \cdots\left(x_{n^{\prime}+m}-x\right)\left(x_{n^{\prime}+m+1}-x\right) \cdots\left(x_{2 n^{\prime}}-x\right)}{(m-1) !\left(n^{\prime}+m\right)\left(n^{\prime}+m-1\right) \cdot x_{n^{\prime}+m+1} \cdots x_{2 n^{\prime}}} \\
& \leq \frac{\left|\left(x-x_{1}\right) \cdots\left(x-x_{n^{\prime}}\right)\right|}{n^{\prime} !} \\
& \times \sum_{m=1}^{n^{\prime}}\left(\frac{n-1}{2}\right)^{n^{\prime}+m} \\
& \times \frac{n^{\prime}\left(x_{n^{\prime}+1}-x\right) \cdots\left(x_{2 n^{\prime}}-x\right)}{(m-1) !\left(n^{\prime}+1\right)\left(n^{\prime}\right) \cdot\left(\frac{2}{n-1}\right)^{n^{\prime}-m}\left(m-\frac{1}{2}\right)(m)(m+1) \cdots\left(n^{\prime}-1\right)} \\
& \leq \frac{\left|\left(x-x_{1}\right) \cdots\left(x-x_{n^{\prime}}\right)\right|}{n^{\prime} !} \cdot n^{\prime} \cdot\left(\frac{n-1}{2}\right)^{2 n^{\prime}} \frac{n^{\prime}\left(x_{n^{\prime}+1}-x\right) \cdots\left(x_{2 n^{\prime}}-x\right)}{\left(n^{\prime}+1\right) n^{\prime} \cdot\left(n^{\prime}-1\right) !\left(1-\frac{1}{2}\right)} \\
& \leq \frac{2\left|\left(x-x_{1}\right) \cdots\left(x-x_{n^{\prime}}\right)\right|}{\left(n^{\prime} !\right)^{2}} \cdot n^{\prime} \cdot\left(\frac{n-1}{2}\right)^{2 n^{\prime}}\left(x_{n^{\prime}+1}-x\right) \cdots\left(x_{2 n^{\prime}}-x\right) \text {. }
\end{aligned}
$$


Hence, from (18) and (19),

$$
\lim _{n \rightarrow \infty}\left|\frac{L_{n}(\phi ; x)}{W_{n}(x)}\right|^{1 / n}=\lim _{n \rightarrow \infty}\left\{\frac{1}{\left(n^{\prime} !\right)^{2}}\left(\frac{n-1}{2}\right)^{2 n^{\prime}}\right\}^{1 / n} .
$$

Finally, by using Stirling's formula

$$
n !=\sqrt{2 \pi n} n^{n} e^{-n}\left(1+w_{n}\right), \quad \lim w_{n}=0,
$$

we can easily verify that the limit in (20) is $e$.

\section{ACKNOWLEDGMENT}

We thank the referee for the comments that improved the presentation of the paper (especially the statement of Corollary 4 and the construction of $x^{*}$ in the remark).

\section{REFERENCES}

[BMS] G. J. Byrne, T. M. Mills, and S. J. Smith, On Lagrange's interpolation with equidistant nodes, Bull. Austral. Math. Soc. 42 (1990), 81-89.

[D] P. J. David, Interpolation and approximation, Blaisdell, New York, 1965.

[GR] I. S. Gradshteyn and I. M. Ryzhik, Tables of integrals, series, and products, Academic Press, New York, 1980.

[HW] G. H. Hardy and E. M. Wright, An introduction to the theory of numbers, 5th ed., Oxford Univ. Press, Oxford, 1979.

[IK] E. Issacson and H. B. Keller, Analysis of numerical methods, Wiley, New York, 1966.

[N] I. P. Natanson, Constructive function theory, Vol. III, Frederick Ungar, New York, 1965.

Department of Mathematics, University of Central Florida, Orlando, Florida 32816 E-mail address, X. Li: fdli@ucf1vm.bitnet 